\title{
Silver Nanoparticle Synthesis and Characterization from Leaf Extract of Psoralea corylifolia (Babchi)
}

\author{
HUMAIRA RANI ${ }^{1 *}$, VIJAY KUMAR SINGH ${ }^{2}$, SHAFAQUE ASIF ${ }^{1}$ and MOHAMMAD ISRAIL ANSAR ${ }^{3}$ \\ ${ }^{1}$ Amity Institute of Biotechnology, Amity University Uttar Pradesh, Lucknow Campus, Lucknow-226 028, India. \\ ${ }^{2}$ Department of Biotechnology, Chhatrapati Shahu Ji Maharaj University Kanpur-208024, India. \\ ${ }^{3}$ Department of Botany, University of Lucknow, Lucknow-226 007, India. \\ ${ }^{*}$ Corresponding author E-mail:humairapricess450@gmail.com
}

http://dx.doi.org/10.13005/ojc/340562

(Received: July 28, 2018; Accepted: September 03, 2018)

\begin{abstract}
Metallic nanoparticles, one of the building blocks of nanotechnology, have a variety of applications because of their unique and interesting properties. In this study, we have done the synthesis of silver nanoparticles (AgNPs) by "bottom-up" technique using an extract of Psoralea corylifolia (Babchi) and silver nitrate $\left(\mathrm{AgNO}_{3}\right)$. The synthesis of AgNPs was monitored by ultravioletvisible absorption spectroscopy. Primary characterization by UV-Vis spectroscopy and scanning electron microscopy (SEM) micrograph depicted that the synthesized nanoparticles of spherical and cubical in shape with uniform size distribution. In addition, the X-ray diffraction (XRD) further revealed that the nanoparticles were of the size ranging from $50-100 \mathrm{~nm}$.
\end{abstract}

Keywords: Nanoparticles, Psoralea corylifolia, Silver nitrate, Scanning electron microscopy (SEM), X-ray diffraction (XRD).

\section{INTRODUCTION}

Psoralea corylifolia is a medicinal plant which develops normally in the blustery season all over India. This plant has antioxidant, antibacterial, anti-fiery and chemo defensive properties. Agreeing to Ayurveda, the entire plant is advantageous to treat huge natural esteem and is been utilized treats quite a while. P. corylifolia comprises of primary segment psoralen and isopsoralen, along these lines lie is antitumor, antibacterial and antiviral properties. This herb purifies the blood and cures blood malignancy.
P. corylifolia is additionally considered as a nerving tonic which fortifies, empowers and quiets the sensory system. In addition, this plant has a local heritage with global importance. It has curative properties due to the presence of various complex chemical substances of different composition, which are found as primary and secondary plant metabolites in one or more parts of the plants ${ }^{1-4}$.

P. corylifolia extract contains a number of chemical compounds which are very important for medicinal purpose, which includes flavonoids

This is an Open Access article licensed under a Creative Commons Attribution-Non Commercial-Share Alike 4.0 International License (https://creativecommons.org/licenses/by-nc-sa/4.0/), which permits unrestricted Non Commercial use, distribution and reproduction in any medium, provided the original work is properly cited. 
(Corylin, Corylifol, Angelicin (neobavaisoflavone, isobavachalcone, bavachalcone, bavachinin, corylifolin and 6-prenylnaringenin, isopsoralenandmeroterpenes (5). A very high concentrations of genistein has been found in the leaves of $P$. corylifolia (6). Genistein is an isoflavonoid and phytoestrogen with antineoplastic, antitumor activity. These plant metabolites according to their composition are grouped as alkaloids, glycosides, corticosteroids, essential oils, etc. Studies have indicated that biomolecules like proteins, phenols, and flavonoids not only play a role in reducing the ions to the nano-size but also play an important role in the capping of the nanoparticles (7). The reduction of $\mathrm{Ag}^{+}$ions by combinations of biomolecules found in these extracts such as vitamins, enzymes, proteins, organic acids such as citrates, amino acids, and polysaccharides ${ }^{7,8}$ are environment-friendly.

Presently, the nano-materials are receiving considerable attention because of their exceptional physicochemical properties over bulk counterparts ${ }^{9}$. Nanotechnology is a field of science with enormous potential in medicine. Nanotechnology plays an extremely significant role in present research as it is the most proficient technology that can be used in almost all the fields such as cosmetics, pharmaceutical, environmental health, electronics, health care, food and feed, chemical industry, biomedical science, energy science, drug and gene delivery, mechanics and space industries. Furthermore, applications of nanotechnology allow the treatments of cancer, allergy, diabetes, inflammation, and infection ${ }^{10}$. The synthesis of nano-materials using chemical methods is very expensive, require complex steps and leads to absorption of toxic chemicals on the surface that may have an adverse effect in the medical application, and are hazardous to the environment. Therefore, development of clean, biocompatible, non-toxic and eco-friendly methods are gaining importance in nanotechnology using microorganisms, enzymes and plant extracts ${ }^{11-14}$. Recently, AgNPs were synthesized using the Acacia concinna fruit extract ${ }^{15}$. Synthesis of AgNPs using the extract of Artemisia nilagirica leaves has been reported by Mathews et al.,"12. SEM and energy-dispersive spectroscopy (EDX) were used to characterize the AgNPs. The morphology of the AgNPs was determined by SEM, and the average diameter of the particles was found to be in the range of 70 to $90 \mathrm{~nm}$. Many investigations suggest several synthesis routes using plant extracts as reducing agents in green synthesis or biosynthesis scheme for AgNPs. Use of extract of Neem (Azadirachta indica L), Acalypha indica, Azadirachta, Emblica and Cinnamomum Emblica officianalis, lemongrass, and other potential plants has been reported $16,15,10$.

In this study, we have synthesized AgNPs exploiting leaf extract of $P$. corylifolia a green reducing material and stabilizing agent. These biogenically synthesized (AgNPs) were characterized using UV-Vis spectroscopy and scanning electron microscopy (SEM).

\section{MATERIALS AND METHOD}

\section{Selection and collection of plant material}

Fresh leaves of $P$. corylifolia were collected from the botanical garden of Chhatrapati Shahu Ji Maharaj University Kanpur, India. Green leaves were taken at flowering stage of the plant.

\section{Preparation of plant leaf extract}

Fresh leaves of $P$. corylifolia were thoroughly washed for 2-3 times with double distilled water (DDW). Leaves were dried in shade at room temperature and cut into small pieces. $2.5 \mathrm{~g} \mathrm{P}$. corylifolia cut leaf were transferred into $250 \mathrm{ml}$ beaker containing $50 \mathrm{ml}$ of distilled water and boiled for about $10 \mathrm{~min}$. at $100^{\circ} \mathrm{C}$. The extract was filtered through Whatman filter paper and the solution was allowed to cool at $4^{\circ} \mathrm{C}$ in $250 \mathrm{ml}$ Erlenmeyer flak for further synthesis of AgNPs.

\section{Biosynthesis of silver nanoparticles}

Silver nitrate (Sigma-Aldrich) serves as a precursor for AgNPs. An aqueous solution (1.0 mM) of silver nitrate was prepared in $250 \mathrm{ml}$ Erlenmeyer flasks and $10 \mathrm{ml}$ of leaf extract and $90 \mathrm{ml}$ of distilled water was added to make the final volume $100 \mathrm{ml}$. Now, qualitatively, AgNPs synthesis was observed as a result of the color transformation from yellow to light brown after $15 \mathrm{~min}$. and further to dark brown after $60 \mathrm{~min}$. incubation under constant stirring at room temperature. No color change was observed in the control samples. The color change in the experimental sample clearly indicates the formation of silver nanoparticles. 


\section{Characterization of silver nanoparticles}

The synthesized AgNPs were monitored by localized surface plasmon resonance (LSPR) using ultraviolet-visible absorption (UV-Vis) spectroscopy (Shimadzu 4650; Model: UV-1800, 240 $\mathrm{V})$ in the range of $200-800 \mathrm{~nm}$. The shape, size and surface of the synthesized AgNPs were analyzed using scanning electron microscopy (SEM) with high-resolution images and selected area, electron X-ray diffraction (XRD) was used for the characterization of synthesized AgNPs. For XRD measurements, a film of NPs was formed on a glass sample holder by drop casting method.

\section{RESULT AND DISCUSSION}

Synthesis and characterization of engineered AgNPs using UV-Vis absorption spectroscopy

Synthesis of bio-engineered was confirmed by UV-Vis absorption spectroscopy. The qualitative study for the green synthesis of AgNPs was associated with visual observations, i.e. the specific color transformation of the reaction mixture of silver nitrate and leaf extract of $P$. corylifolia. The formation of AgNPs was visualized with the color change from yellow to dark brown. This characteristic color variation is due to the UV-Vis absorption spectroscopy emerged as a valuable tool to characterize as well as validate the synthesis and stability of nanoparticles in an aqueous suspension, where the nano-particle of silver reveals absorption in the range of $420 \mathrm{~nm}$. The surface plasmon resonance (SPR) absorption spectra of nanoparticles are mainly dependent on particle size, stabilizing molecules, state of aggregation and the dielectric constant of the medium ${ }^{17}$. It shows an absorbance peak at $420 \mathrm{~nm}$ (Fig. 1) which is specific for silver nanoparticles.

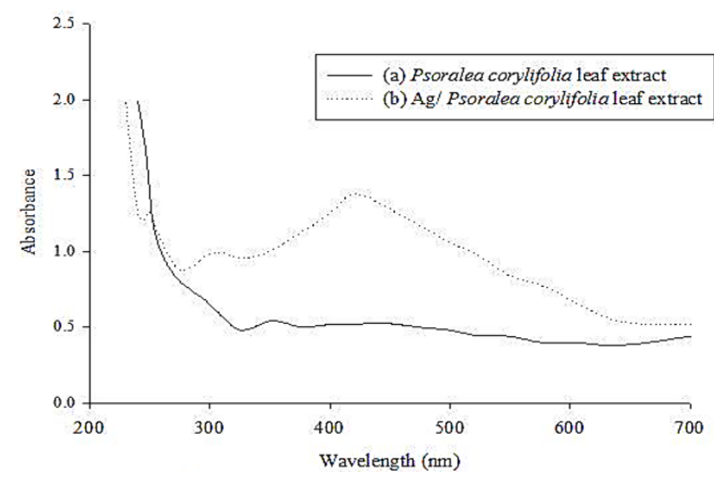

Fig. 1. UV-Vis absorption spectra of (a) Psoralea corylifolia (Babchi) leaf extract and (b) Ag/P. corylifolia (Babchi) emulsion after $60 \mathrm{~min}$. of incubation

\section{X-Ray Diffraction analysis}

The XRD curve confirmed the formation of silver nanoparticles (Fig. 2). Interpretation of AgNPs XRD pattern reveals the existence of diffraction lines at low angles $\left(20^{\circ}\right.$ to $\left.70^{\circ}\right)$. The silver nanoparticles showed the peaks of silver at $2 \theta=32.130^{\circ}, 38.02^{\circ}$, $44.203^{\circ}$ and 64.439 that can be assigned to the (111), (200), (211) and (311) facets of silver, respectively, which go very well with the values manipulated for face centered cubic structure of silver nanocrystals.

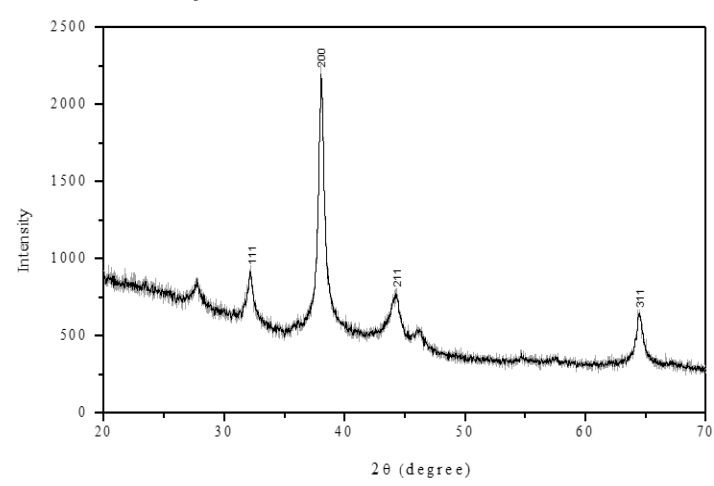

Fig. 2. XRD pattern of biogenic synthesized AgNPs of P. corylifolia (Babchi) leaf extract

Scanning electron microscopic(SEM) analysis

Scanning electron microscopy was done for revealing the surface morphology of particles. The results of SEM analysis showed that the silver nanoparticles synthesized are of uniform size, distributed evenly and spherical/cubic in shape ranging 50-100 $\mathrm{nm}$ (Fig. 3). Other groups of researchers have also reported the synthesis of nanoparticles from various plant sources, that the silver nanoparticles obtained from various sources are mostly nanosphere with some nanorods in shape and different sizes ${ }^{18-20}$.

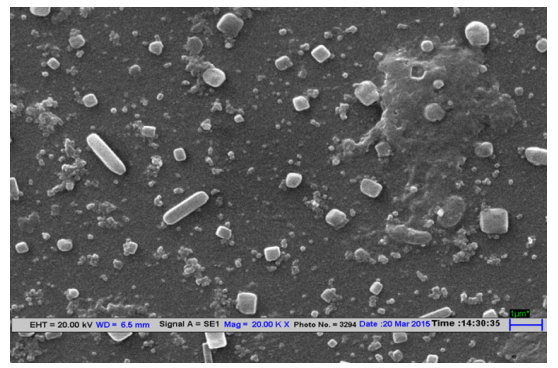

Fig. 3. SEM image of synthesized AgNPs from P. corylifolia (Babchi) leaf extract

\section{CONCLUSION}

A green novel route for the rapid, without using any chemical reagent or surfactant template 
was used in this approach that consequently allows the bioprocess with the benefit of being environmentally friendly and non-toxic biosynthesis of AgNPs at room temperature using easily available $P$. corylifolia leaf extract which acts as a green reducing as well as a stabilizing agent.

\section{ACKNOWLEDGEMENT}

Authors are grateful to the Head, Department of Biotechnology, Chhatrapati Shahu Ji Maharaj University Kanpur, India, for providing the facilities to carry out research work.

\section{REFERENCES}

1. Asmita, J.; Gavhane, I.; Padmanabhan, P.; Suresh, P.; Kamble and Suresh N. Int. J. Pharm. Bio. Sci., 2012, 3(3), 88-100.

2. Geethalakshmi, R. and Sarada. D. V. L. Int. J. Eng. Sci and Tech., 2010, 2(5), 970.

3. Bar, H.; Bhui, K.D.; Sahoo, P. G.; De, P. Sarkar.; Misra, Ajay. A. Physicochem. Eng. Asp., 2009, 339, 134-139.

4. Venkataraman, D.; Kalimuthu, K.; Sureshbabu, R.K.P. and Sangiliyandi, G. Met. Nano in Mic., 2011, 2 , 173-214.

5. Zhao, L.H.; Huang, C.Y.; Shan, Z.; Xiang, B.G.; Mei, L.H. J.Chromatogr. Bio., 2005, 82, 67-74.

6. Kaufman, P.B.; Duke, J.A.; Brielmann, H.; Boik, J.; Hoyt, J.E. J. Alt Compl Medi., 1997, 3(1), 7-12.

7. Naheed, A.; Seema, S.; Singh, V. N.; Shamsi, S.F.; Anjum, F. and Mehta, B. R. Bio. Res. Intl., 2011, 8, 5241-5246.

8. Yao, Z.;Wenshuang, L.; Jiale, H.;Wenta, W.;Yixian, G.; Liqin, L.; Qingbiao, L.; Ling, L. and Mingming, D. Nano. Res. Lett., 2010, 51, 351-1359.

9. Jha, K.A.; Prasad, K.; Kumar,V. Biotec. Prog., 2009, 25, 1476-1479.

10. Uber, A.; Naveed, S.; Ramzan, N.; Rafique, S.M. Worl. Scie. Nano., 2012, 07(5), 30005.

11. Jiale, H.; Qingbiao, L.; Daohua, S.; Yinghua,
L.;Yuanbo, S.; Xin, Y.; Huixuan, W.; Yuanpeng, W.; Wenyao, S.; Ning, H.; Jinqing, Hand.; Cuixue, C. Nanotechnology., 2007, 18, 105104-105115.

12. Matthew, A.A.; Cameron, W.E. and Colin, L. R. Green.Chem., 2006, 8, 417-432.

13. Mansour, G.M.; Robabeh, H.D. J. Ind. Eng. Chem., 2014, 20, 739.

14. Torresdey, J.L.G.; Gomez, E.; Videa, J.R.P.; Parsons, J.G.; Troiani, H.; Yacaman, M. J. Langmuir., 2003, 19, 1357-1361.

15. Krishnaraj, E. G.; Jagan, S.; Rajasekar, P.; Selvakumar, P. T.; Kalaichelvan, M. N. Colloids. Surf. B., 2010, 76, 50.

16. Shankar, S.S.; Ahmad, A.; Sastry, M. Biotechnol. Prog., 2003, 19, 1627-1631.

17. Kasthuri, J.; Veerapandian, S. and Rajendiran, N. Colloids. Surf. B. Biointerface., 2009, 68, 55-60.

18. Nestor, A.R.V.; Mendieta,V.S.; Lopez, M.A.C.; Espinosa, R. M. G. and Alatorre. J. Mater. Let., 2008, 62, 3103-31053.

19. Meena, R.K.; Ansari, K.; Kishore, N. and Chouhan, N. Res. J. Rec. Sci., 2018, 7, 43-47.

20. Veronica. A.; Isaac, H.; Jose, R. P.V.; Miguel, J.Y.; Horacio, T.; Patricia, S.; Jorge, L. G. T. J. Nanopart. Res., 2004, 6, 377-382. 